\title{
Las grandes líneas tendenciales del proceso civil a fines del segundo milenio
}

Roberto Omar Berizonce

1. Irrupción y gloria de la sistemática "constructivista"; su trascendencia.

El siglo que fenece fue testigo del nacimiento del Derecho Procesal sistemático, que desde sus albores en 1903 cuando Chiovenda formula su famosa prolusión en el curso del Bologna, $L$ 'azione nei sistema dei diritti, transitó un largo camino de afirmación y desenvolvimiento que llevaron a instalar, en el pensamiento doctrinal primero y prontamente en las instituciones legales, toda una concepción sistemática y científica, sostenida en los principios fundantes -la "trilogía estructural"- de jurisdicción, acción y proceso. El pensamiento chiovendiano -de reconocidas raíces germánicas- planteó un profundo cambio conceptual que hacía emerger problemas nunca antes observados, y demostró la autonomía de la ciencia del proceso, instancia superadora del procedimentalismo y del método de la exégesis. La generosa acogida y los tempranos entusiasmos que despertara la naciente disciplina, pervivieron al impulso vivificador que transmitían las renovadas y cada vez más profundas y complejas enseñanzas de los continuadores. Carnelutti aporta la grandiosidad de su sistema, mientras Redenti desarrolla la visión histórica de las instituciones procesales y, a su tiempo, Calamandrei posa su aguda visión en la acción humana en el gran drama judiciario, ve desfilar a partes, jueces y abogados en su doliente humanidad. Todos ellos, como destacara Satta, fueron en el fondo, en un sentido u otro, verdaderos poetas apasionados que idealizaron los resultados de la ciencia. Lo decisivo es la profunda renovación del método de estudio que inauguraron, no sólo para la propia disciplina 
sino con fuerza expansiva y utilidad en todas las ramas del derecho $y$, particularmente, en la teoría general. Al punto que se ha afirmado -no sin cierta exageración- que el advenimiento del derecho procesal civil fue el hecho más saliente en la historia de la ciencia jurídica del siglo ${ }^{1}$.

En el desenvolvimiento de aquella clásica trilogía, la concepción dualística derecho sustancial-acción fue dejando paso y superada a partir de una renovada visión unitaria del fenómeno jurídico. No puede pensarse en el derecho sustancial como existente y operante, allí donde es negado en su concreta realización. La acción, se afirma, es en realidad el derecho, el único derecho que se puede concretamente concebir, y tiene por su contenido la postulación en juicio. El fulcro del derecho procesal se desplaza desde la ley al juez, o mejor, que ley y juez son los dos inescindibles momentos de la concreción del derecho, que está en poder de la parte ${ }^{2}$.

\section{El tránsito superador (desde la plataforma de los logros de la sis- temática) hacia una visión totalizadora de los fenómenos del pro- ceso y del sistema de justicia.}

De ahí en más es todo historia reciente. Mauro Cappelletti ${ }^{3}$, el dilecto discípulo florentino de Calamandrei, anunciaba en 1972 en el prólogo

1 Hemos seguido en esta descripción a Satta S., "La dottrina del Diritto Processuale Civile", Riv. Dir. Proc., 1992, n³, p.703, ensayo de 1974 que fuera su último trabajo, según lo anota alli Cipriani. La historia del procesalismo moderno durante el lapso de referencia es, por otra parte, ampliamente conocida. Entre nosotros, debemos especialmente su difusión como en tantas otras materias- a Sentis Melendo (Estudios de Derecho Procesal, EJEA, Bs.As., 1967, v.II, passim). El siempre recordado maestro español destacó también la profunda incidencia de las ideas de la doctrina clásica italiana entre los cultores argentinos: "50 años de proceso civil argentino ¿̨donde estamos?”, en su obra póstuma, La prueba. Los grandes temas del derecho probatorio, EJEA, Bs.As., 1979, p.461.

2 Satta S., op. cit., p.709. No es diferente la perspectiva que el tan cercano maestro Mercader desarrollara en sus últimos escritos. Así, en Estudios de Derecho Procesal, Ed. Platense, 1964, pp. 326; id., El proceso y la unidad del orden jurídico, J.A., 1968-III, doct., p.744. Conf.: A.M. Morello, Mercader en la cultura procesal argentina, J.A., 1967-VI, p.839; id., "El proceso en el ordenamiento jurídico", La Ley, 1991-C, p.831 y también en Estudios de Derecho Procesal, LEP, 1998, v.1, p.3.

3 Debemos también a Sentis Melendo la difusión entre nosotros de la obra de Cappelletti y Denti. Desde la traducción del temprano estudio del entonces profesor de Florencia, Studio del Diritto e tirocinio proffesionale in Italia e in Germania. La crisi delle professioni giudiziarie 
a su Giustizia e Societá los fundamentos de una renovada ciencia del proceso, uno de cuyos primeros frutos veía en la obra de V. Denti, Processo civile e giustizia sociale, de $1971^{4}$. Reflejaba el amanecer de una nueva escuela de pensamiento procesalístico y jurídico en general, entre cuyos precursores se reconocía a Calamandrei y al gran comparatista Ascarelli, que apoyándose en la aplicación de métodos empírico-deductivo e histórico-comparativo, pretende recrear los grandes temas del proceso, insertándolos en nuevas y ampliadas dimensiones -constitucional, social, transnacional- para formular propuestas siempre comprometidas, para la transformación evolutiva de las instituciones jurisdiccionales y su adecuación a las nuevas circunstancias de la sociedad.

En esa visión, el proceso común civil es el espejo donde se reflejan de la manera más directa los conflictos de nuestra época, que tocan el "corazón", la estructura misma de la sociedad. De ahí que el fenómeno, y aun todo el sistema de justicia, ha de ser contemplado necesariamente bajo el perfil de su efectividad, de su accesibilidad, de su adecuación a las instancias de una sociedad renovada y sustentada en valores proyectados hacia la construcción de un orden social más justo. Así, pasan a un primer plano, para convertirse en objeto de análisis y propuestas, temas antes largamente descuidados por la dogmática tradicional, como la eficiencia de la "máquina de justicia", la duración y costo del proceso, la accesibilidad de la justicia a todos ${ }^{5}$, la correspondencia del dere-

in Italia. Problemi e rimedi, Dott. A. Giuffré ed., Milano, 1957, publicada por EJEA en 1959, en traducción de S. Sentis Melendo y M. Ayerra Redin, bajo el tírulo Estudio del derecho y tirocinio profesional en Italia y en Alemania. Luego sucesivamente, diversos estudios agrupados bajo el título La oralidad y las pruebas en el proceso civil, también en traducción de Sentis Melendo (EJEA, Bs.As., 1972), donde se incluye un capítulo de la obra fundamental La testimonianza della parte nel sistema della oralitá. Sigue Principi fondamentali e tendenze evolutive del processo civile nel diritto comparato (1968), bajo el título El proceso civil en el derecho comparado (EJEA, Bs.As., 1973); y, por último, diversos estudios aparecidos originariamente en Processo e ideologie (1969) y en Giustizia e Societá (1972), editados por el mismo sello editorial en 1974: Procesos, ideologías, sociedad (trad. S. Sentis Melendo y T.A. Banzhaf). Además de la traducción y publicación de otros estudios cortos en la colección de Breviarios. Bien podía afirmar el maestro español en la presentación de 1974 que la obra de Cappelletti por entonces estaba en gran parte en nuestro idioma.

4 V. Denti, Processo civile e giustizia sociale, Edizioni di Comunitá, Milano, 1971. Ulteriormente, en Un progetto per la giustizia civile, Il Mulino, Bologna, 1982, passim; id., La giustizia civile. Lezioni introduttive, Il Mulino, Bologna, 1989, passim.

5 La idea del acceso a la justicia ha sido, sin duda, la bandera más significativa del movimiento de reformas por la amplitud de sus propuestas y la acogida que tuvo universal- 
cho procesal a valores de libertad individual y de justicia social, a las exigencias y tendencias evolutivas que se expresan en las nuevas "ideologías" sociales, constitucionales, supranacionales y comunitarias del mundo contemporáneo.

\section{a. La renovación metodológica (factual approach, análisis histórico- comparativo).}

El enfoque metodológico pretende superar la dogmática tradicional, llevando la indagación a la denominada "política del derecho", es decir a las implicaciones sociales, ideológicas y lato sensu políticas del derecho y de las normas e instituciones ${ }^{6}$. En esa perspectiva, se resaltan los fundamentos metajurídicos -ideológicos- de las normas y de su interpretación, con el "compromiso" metodológico que asienta en el cuestionamiento frontal del tradicional método de estudio típicamente escolástico, dogmático y formalístico, que persigue la búsqueda de una ciencia "pura" e ideológicamente "neutral" . En contraposición, se pro-

mente. Infra nota 16. Asimismo: M. Cappelletti, $L$ accesso alla giustizia e la responsabilitá del gitrista nella nostra epoca, en Studi in onere di Vittorio Denti, Cedam, Padova, 1994, v.I, p.263. Para Denti, el aparato garantizador de justicia, característico de los Estados modernos, tienen un significado y un valor solamente para aquellos sectores que son naturales destinatarios del orden a cuyo mantenimiento el proceso, que es una forma de alienación en el sentido marxista, está establecido. Contra esa realidad se enfrentan las tentativas que apuntan a resolver el problema del acceso a la justicia en el mundo contemporáneo, que inicialmente se ha presentado en dos flancos especialmente sensibles: la asistencia jurídica a los no habientes y la tutela de los intereses colectivos (Un progetto..., op. cit., pp.125-128; 161 y ss.; 187 y ss.).

6 M. Cappelletti, Proceso, ideologías, sociedad, op. cit., Introducción, p. IX-XVII; id., "Algunas reflexiones sobre el rol de los estudios procesales en la actualidad", Jus, Rev. Juríd., La Plata, 1988, ${ }^{\circ} 39$, p.6; id., L' accesso alla gitistizia e la responsabilitá.., op. cit., pp.265-271. Conf. allí la referencia a Denti V., Sistematica e post-sistematica nella cvoluzione delle dottrine del processo, en Riv, critica del diritto privato, 1986, pp.469-482. Como afirmaba Calamandrei, el trabajo de los estudiosos es hecho "no para servir el gusto arquitectónico de las abstractas conclusiones sistemáticas, sino para servir en concreto a la justicia". De ahí que la necesidad de dar un nuevo curso a los estudios sobre la justicia civil, a través de propuestas comprometidas con tal exigencia (Denti V., Un progetto..., op. cit., p.11). Para la evaluación de los estudios procesales en Alemania, en el mismo sentido, a partir del "constructivismo" sistemático de la pandectística y las actuales tendencias superadoras: Walter G., Cinquanta anni di studi sul processo civile in Germania: dal costnuctivismo all'apertura internazionale, Riv. Dir. Proc., 1998, n 1 , pp.36 y ss.

7 El dogmatismo jurídico -expresa Cappelletti- ha sido una forma degenerativa del positivismo jurídico, que ha llevado no solamente a una simplificación irreal del derecho, reducido a su aspecto normativo -izts positum- descuidándose así los otros no menos esenciales 
pugna una concepción del derecho como fenómeno social y, en consecuencia, la ciencia jurídica como ciencia sociológico-valorativa; una ciencia de problemas prácticos y no de sistematizaciones conceptuales abstractas, de resultados concretos y no de deducciones apriorísticas, en fin de elecciones creativas y responsables y no de conclusiones automáticas. Los objetos de la indagación se afrontan no con el método abstracto-deductivo del iusnaturalismo tradicional, sino con el método realístico-inductivo superador de las "doctrinas de las geometrías" -como las denominaba Calamandrei- y de la pretendida pero mistificadora "neutralidad" o "pureza" de sus contenidos, en la que radica precisamente la ideología de la aceptación y de la conservación, que cuando se desentiende de las exigencias de su tiempo ya no es orden sino reacción.

\section{b. La política judicial (y del derecho en general), los valores fundantes y el compromiso de los juristas.}

El método de pensamiento y análisis jurídico no se limita, todavía, a problematizar las normas ${ }^{8}$ e instituciones y su modo de operar, como meritoriamente lo han hecho las varias corrientes del pensamiento realista moderno 9 , sino que introduce un cambio fundamental en la óptica de la contemplación de esos objetos. La traslada desde la perspectiva tradicional de los "productores" del sistema jurídico -jueces, abo-

elementos: sujetos, instituciones, procedimientos; pero ha llevado además a una igualmente perniciosa simplificación de los deberes y de las responsabilidades del jurista ( $L^{*}$ accesso alla giustizia e la responsabilitá ..., op. cit., p.265).

8 El aspecto normativo del derecho no está relegado, pero es visto como uno de los elementos, y ni siquiera el más importante, dado que preeminente son las personas (con todas sus peculiaridades culturales, económicas, sociales), las instituciones, los procesos, a través de los cuales el derecho vive, se forma, se desenvuelve y se impone. Además, el derecho está visto no ya como sistema separado, autónomo, sino como parte integrante de un más complejo ordenamiento social, donde no puede ser artificialmente aislado de la economía, de la moral, de la política. Es la concepción "contextual" del derecho (M. Cappelletri, $L$ 'accesso a la giustizia e la responsabilitá ..., op. cit., p.270).

9 Las corrientes de pensamiento conocidas como "jurisprudencia de intereses" y "realismo jurídico", han rebatido el conceptualismo abstracto y formalístico de la jurisprudencia dogmática. Sin embargo, el realismo jurídico ha desembocado en el llamado "excepticismo normativo", rraducido a menudo en escuelas de pensamiento exageradas - "deconstruction del derecho", total indeterminacy, critical legal studies, law and economics (M. Cappelletti, L'acceso alla giustizia e la responsabilitá..., op. cit., pp.266-269, con amplia bibliografía). 
gados- a la de los "consumidores", usuarios, y sus consecuentes demandas y reclamos, que es la que mejor consulta las exigencias de una sociedad democrática, libre y abierta, en la que los productores y los servicios deben organizarse con el objetivo de satisfacerlas. Lo cual es como decir que el derecho y el Estado deben ser finalmente vistos como lo que son: como simples instrumentos al servicio de los ciudadanos y de sus necesidades, y no a la inversa ${ }^{10}$.

El jurista tiene el deber científico a más de moral de centrar su labor investigadora en las exigencias sociales y materiales de su época, máxime en tiempos como los nuestros de aguda crisis, incertidumbres y angustias, en los cuales los valores supremos vuelven a estar en discusión, y que por ello mismo se patentiza dramáticamente la necesidad de llegar al fondo de la cosas, calando hondo y aguzando el ingenio y la imaginación en la búsqueda de las propuestas adecuadas, para la construcción de un sistema nuevo más realista y más humano ${ }^{11}$. Es en este terreno donde se enfatiza la importancia decisiva del análisis comparativo -método fenomenológico comparativo- ${ }^{12}$.

\section{Las grandes tendencias evolutivas del derecho y la justicia: consti- tucional, social, transnacional.}

El análisis bajo ese prisma de las grandes tendencias evolutivas de los

10 M. Cappelletti, "Acceso a la Justicia (como programa de reformas y como método de pensamiento)", Rev. Col. Abog. La Plata, 1981, N41, pp.167-170, trad. G. Seminara.

11 M. Cappelletti. Proceso, ideologías, sociedad, cit., Introducción, pp.IX-XII. La teoría no es sino estéril documentación -o crociana "crónica, no historia"- si no se convierte ella misma en matriz de acción: los juristas, como los filósofos de Marx, no tienen sólamente la tarea de interpretar el mundo, sino también la de transformarlo (...). El jurista verdadero es el que coloca su investigación al servicio de la solución de los problemas concretos (op. cit., p.XIV). Conf: V. Denti. Un progetto..., op. cit., passim.

12 El método comparativo se atiene a la realidad de los datos y ofrece, no obstante, la posibilidad de una valoración no meramente subjetiva de las soluciones adoptadas por los varios ordenamientos, a la luz de la objetiva eficacia en la resolución de los problemas sociales en vista de los cuales han sido proyectados (M. Cappelletti. Ĺaccesso a la giustizia e la responsabilitá..., op. cit., p.272). Las finalidades que persigue el comparatista se reducen a tres: a) la comparación como instrumento de politica del derecho; b) como investigación de historia del derecho; y c) como análisis estructural del derecho (V. Denti. Un progetto..., op. cit., pp.23 y ss.. Conf. G. Walter. op. cit., donde se destaca la evolución de la doctrina procesal alemana que, superando la comparación "constructivista" sistemática, derivó hacia la utilización como método e instrumento de la políicica del derecho (p.50). 
ordenamientos jurídicos contemporáneos, revela tres fundamentales movimientos de acción y de pensamiento, que constituyen otras tantas "dimensiones" del derecho y la justicia ${ }^{13}$. En primer lugar, la dimensión constitucional, empeñada en la búsqueda de ciertos valores fundamentales que emergen crecientemente con fuerza de lex superior, a través de procedimientos y jurisdicciones constitucionales ampliamente difundidos desde el fin de la última gran conflagración mundial ${ }^{14}$. En paralelo, la dimensión transnacional, que implica la tentativa de superar los rígidos criterios de las soberanías nacionales, a partir de la creación de diversos núcleos de una lex universalis (declaraciones de derechos, bill of Rights, tendencialmente universales) y una jurisdicción transnacional ${ }^{15}$. Finalmente, la dimensión social del derecho y la justicia, resumida en el programa del acceso a la justicia ${ }^{16}$. La dimensión

13 M. Cappelletti. "Appunti per una fenomenologia della gitustizia nel XX secolo", en Studi in on. di E.T. Liebman, Milano, Giuffré, 1979, v.I, p.153 y ss.; id., "Fundamental Guarantees of the Parties in Civil Litigation: Comparative Constitutional, International, and Social Trends", en 25 Stanford Law Rev., 1973, pp.651 y ss., 715; id., Acceso a la justicia (como programa de reformas...), op. cit., pp.153-158.

$14 \mathrm{M}$. Cappelletti. Jurisdicción constitucional de la libertad, Imprenta Universitaria, México, 1961, trad. H. Fix Zamudio; id., en Proceso, ideologías, sociedad, op. cit., p.453; id., El control judicial de la constitucionalidad de las leyes en el derecho comparado, UNAM, México, 1966, trad. C. Gómez Lara y H. Fix Zamudio; id., La Justicia Constitucional (estudios de derecho comparado), U.N.A.M., México, 1987, que incluye varios estudios sobre el tema.

15 M. Cappelletti. "Justicia constitucional supranacional", en La justicia constitucional..., op. cit. p.213, trad. L. Dorantes Tamayo; id., L'acceso alla giustizia e la responsabilita.., op. cit., pp.289-292. Entre sus últimos aportes: "Evolución de Europa". Anales de la Academia Nacional de Derecho y Ciencias Sociales de Buenos Aires, 2a. época, n³1, 1993 (anticipo), trad. G. Tempesta.

En el trámite hacia la apertura internacional del proceso común, los objetivos se centran al presente en la elaboración de las líneas fundamentales de un ius comune de un derecho procesal universal, sustentado en la coordinación y la armonización de las legislaciones nacionales. Empeño grandioso que convoca en estos tiempos a los procesalistas. Conf.: G.Walter. op. cit., p. 52. Sobre los recientes proyectos "Storme" y "Hazard-Taruffo", éste último concebido como "un código de proceso civil mundial", puede verse del mismo profesor de la Universidad de Berna (G. Walter.): "Aspetti internazionali del diritto processuale", Riv. Trim. Dir. Proc. Civ., 1996, pp. 1162 y ss..

16 Es ampliamente difundida la vasta investigación conocida como Proyecto de Florencia, dirigida por Cappelletti, que rematara en la publicación de cuatro volúmenes en seis tomos, y en la cual participaran un centenar de expertos (juristas, sociólogos, economistas, antropólogos, polirólogos y psicólogos) de los cinco continentes: M. Cappelletti (gen. editor), The Florence Access to Justice Project. vol.I (Books I and II), M. Cappelletti y B. Garth (eds.), Access to justice: A world survey, Milano y Alphen aan den Rijn (Holand), 1978; vol.II (Books I and II), 
social representa la tentativa de responder al problema y la crisis derivadas de las profundas transformaciones de las sociedades industriales y post-industriales modernas, en las cuales la petición de justicia adquiere un sentido cada vez más decididamente de petición de igualdad no sólo formal, sino real y efectiva igualdad de posibilidades. El problema del acceso se presenta bajo dos aspectos principales: por un lado, como efectividad de los derechos sociales, que no tienen que quedar en el nivel de declaraciones meramente teóricas sino que deben efectivamente influir en la situación económico-social de los miembros de la sociedad; por otra parte, como búsqueda de formas y de métodos, a menudo nuevos y alternativos a los tradicionales, por la racionalización y el control del aparato gubernamental y por consiguiente para la protección contra los abusos a que el mismo puede dar lugar, directa o indirectamente ${ }^{17}$. En una primera etapa se centró el interés en el debate sobre los obstáculos económicos, los costos del proceso y el sistema de asistencia jurídica a los pobres ${ }^{18}$; luego se focalizó la defensa de los intereses colectivos o difusos ${ }^{19}$, para superar los obstáculos organizativos

M. Cappelletti y J. Weisner (eds.), Access to Justice: Promising Institutions, Milano y Alphen aan den Rijn, 1978-79; vol. III, M. Cappelletti y B. Garth (eds.), Access to Justice: Emerging Issues and Perspectives, Milano y Alphenaan den Rijn, 1979, vol. IV, K. F. Koch (ed.), Access to Justice: The Anthropological Perspective, Milano y Alphen aan den Rijn, 1979. Una síntesis comparativa está constituida por la relación general de Cappelletti y Garth, publicada en vol. I, Book I, pp.1-124 con el título Access to Justice: The Worlwide Movement to Make Rights Effective. La primer versión castellana de ésta última, traducción de S. Amaral, fue El acceso a la Justicia, ed. Col. Abog. La Plata, 1983, con presentación de R. O. Berizonce. Complementariamente el volumen M. Cappelletti (ed.), Access to Justice and the Welfare State, Alphen aan den Rijn Bruxelles-Stuttgart-Firenze, 1981, recoge las relaciones de algunos eminentes expertos europeos y norteamericanos sobre las tres grandes "oleadas" del movimiento de reforma por el acceso a la justicia, con su evaluación de los resultados de toda la investigación. Un amplísimo catálogo de obras recordadas por su contribución a estas ideas se incluye en la referida monografía de Cappelletti, El acceso a la Justicia (como programa de reformas...), op. cit., pp.155-158.

17 M. Cappelletti M., Acceso a la reforma (como programa de reformas...), op. cit., pp.159-160; id., L'accesso alla giustizia e la responsabilitá.., op. cit., pp.273-274.

18 M. Cappelletti y B. Garth., El acceso a la justicia, op. cit., pp.39-58. V. Denti. "Accesibility of Legal Procedures for the underprivileged: Legal Aid and Advice", en Towars a Justice with a Human Face, M. Storme and Casman ed., Antwerpen/Deventer, Kluwer, 1978, p.168; id., Processo civile e giustizia sociale, op. cit., p.31-52; id., Un progetto..., op. cit., pp. 133-159; 161-185.

19 M. Cappelletti y B. Garth, ob. cit., pp.58-84; M. Cappelletti, L'accesso alla giustizia e la responsabilitá..., op. cit., pp.278-283. Los aportes de Denti fueron igualmente trascendentes: 
especialmente en relación a los consumidores y usuarios; $\mathrm{y}$, por último, como concreción de todo lo anterior, la concepción de un vasto programa de reformas del funcionamiento del sistema judicial y más genéricamente del aparato de justicia ${ }^{20}$. Empeño de grandiosas proyecciones entre cuyos fines, entre otros, se destacan: 1) el de adoptar procedimientos más accesibles en cuanto más simples y racionales, más económicos, eficientes y especializados para cierto tipo de controversias; 2) el de promover y hacer accesible un tipo de justicia diferenciada, definido como "coexistencial"; 3) el de someter la actividad pública a formas, a menudo nuevas y de cualquier manera más ensanchadas y accesibles, de control y, en general, de crear formas de justicia más accesibles en cuanto más descentralizadas y "participatorias"21. Es así como se estudian los medios alternativos para la resolución de conflictos, los procedimientos y órganos especiales para atender litigios de particular "importancia social", tribunales para resolver asuntos de menor cuantía y de vecindad, para las demandas de los consumidores, la tutela del ambiente, las transformaciones en la condición de los operadores jueces (jueces legos, auxiliares "cuasiprofesionales"), abogados especializados, etc ${ }^{22}$.

\section{Proyecciones y perspectivas al cierre del milenio.}

El vastísimo programa propuesto, que hemos dejado tan sólo esbozado, con el rico repertorio de las ideas que le acuerdan sustento y los requerimientos sociales igualmente complejos y variables a los que se brinda respuesta desde el movimiento universalmente conocido como el "acceso a la justicia", han servido y alentado en las últimas tres décadas innumerables avances en el plano de la teoría procesal en general y aún en otras diversas disciplinas jurídicas, como el derecho constitucional y el derecho internacional privado- y especialmemente, en el diseño de las políticas judiciales y su concreta implementación.

20 M. Cappelletti y B. Garth, ob. cit., pp.84 y ss.. M. Cappelletti, L'accesso alla giustizia e la responsabilitá.., op. cit., pp.283-286.

21 M. Cappelletti, Acceso a la Justicia (como programa de reforma...), op. cit., pp.184187.

22 M. Cappelletti y B. Garth., op. cit., pp.91-98; 101-105; 105-126; 126-134; 134158; 162-164. M.Cappelletti, L'accesso alla giustizia e la responsabilitá.., op. cit., pp.283. 
Sería ilusorio el intento de cualquier inventario, siquiera parcial y siempre subjetivamente estrecho, de tales adelantos y logros. Lo que se ha verificado de modo objetivo es la amplia adhesión que recibiera en el mundo jurídico contemporáneo y la receptividad que, en medida no desdeñable, ha derivado de la implementación, en las distintas latitudes, de muchas de las reformas propuestas ${ }^{23}$.

De todos modos ilustran acabadamente sobre las proyecciones en el terreno teórico de la ciencia procesal, ahora con ensanchados contenidos y extendidas fronteras, los laboreos de los cultores de la disciplina volcados en debates y discusiones en los encuentros, jornadas y congresos internacionales celebrados en los últimos cinco lustros. Particularmente, a partir del que tuviera por escenario a Gante (Bélgica, 1977), y en los sucesivos de Würzburg (Alemania, 1983), Utrecht (Holanda, 1987), Coimbra-Lisboa (Portugal, 1991) y Taormina (Italia, 1995), sin olvidar el congreso extraordinario de Bologna (Italia, 1988). Un ligero recorrido por las temáticas de cada uno de esos significativos encuentros, resulta suficiente para avizorar cuáles son las banderas, ideas-fuerza emblemáticas y cuestiones que acaparan el interés de los cultores de la disciplina en los tiempos finiseculares. Ya la convocatoria de Gante contenía una definición valorativa que marcaba un rumbo y una postura jurídica y ética: Towards a Justice with a Human Face (Hacia una Justicia de rostro más humano) ${ }^{24}$. La humanización del proceso ${ }^{25}$ fue uno de los capítulos centrales de las discusiones. El siguiente encuentro de Würzburg estuvo dedicado a la efectividad de la protección judicial y del orden constitucional; bajo el primer aspecto se examinaron tópicos como la organización de los servicios de asistencia jurídica para los carentes de recursos, el derecho a la prueba y la pericia técnico-científica, la conciliación, los procedimientos no contenciosos, la independencia judicial, los conflictos y tribunales de familia. También vinculado con la efectividad de la tutela jurisdiccional de los nuevos derechos, se estudiaron las diversas formas de protección de los intereses

23 La propia investigación constituída por el denominado Proyecto de Florencia, de mediados de los 70 y las evaluaciones y trabajos posteriores, mencionados supra en nota 16 , dan cuenta de los múltiples avances en la concreción de las ideas maestras.

24 Towards a Justice with a Human Face, edit. M. Storme y H. Casman, Antwerpen/ Deventer, Kluwer, 1978.

25 La relación general sobre el tema estuvo a cargo del Prof. de Valencia, V. Fairen Guillén, La humanización del proceso, op. cit., pp.186 y ss.. 
colectivos o difusos, en conexión con la dimensión social. Sin descuidarse los enfoques metodológicos, a través del estudio de la contribución de la sociología del derecho a la reforma procesal ${ }^{26}$. En 1987 la convocatoria se hizo bajo el lema Justicia y eficiencia, para tematizar el más efectivo acceso a los tribunales, en el marco de la crisis del Estado social; así, a través de nuevos mecanismos o procedimientos de solución de los conflictos, v. gr., el arbitraje, las vías administrativas, las alternativas informales, el managing en la administración de la sobrecarga tribunalicia ${ }^{27}$. En la cita ulterior (Portugal, 1991) se focalizó el papel y organización de los magistrados y abogados en las sociedades contemporáneas, debatiéndose entre otros tópicos la organización, independencia y responsabilidad de los jueces, la ética profesional, los poderes del juez y el "activismo" judicial, el entrenamiento y capacitación de unos y otros, al igual que otras cuestiones relativas a la organización de los procedimientos, tales como la participación de los legos, los procesos complejos, el razonamiento en la formación de la sentencia colegiada ${ }^{28}$. Finalmente, el congreso internacional de Taormina estuvo dedicado a los aspectos transnacionales de la ley procesal. La próxima convocatoria, la última de este siglo, en Viena, Austria (1999) anuncia bajo el lema la ley procesal en los albores del nuevo milenio, entre otros tópicos los referidos a la aplicación de los avances tecnológicos en el proceso civil, reducción de la duración y los costos del proceso, armonización y uniformación de la ley procesal, tendencias recientes en la posición del juez y del abogado, la educación legal. Los estudiosos de la moderna ciencia procesal afrontan y están avocados, de ese modo, universalmente, a brindar respuesta a los desafíos siempre latentes de estructurar en los moldes teórico-dogmáticos y sobremanera en su concreción práctica, las transformaciones que el sistema de justicia exige ${ }^{29}$.

26 Effektiver Rechtsschutz und verfassungsmäBige Ordnung, ed. W. J. Habscheid, Gieseking-Verlag, Bielefeld, 1983.

27 Justice and Efficiency, VIII World Congress on Procedural Law, Kluwer, 1988. M. Cappelletti, Algunas reflexiones..., op. cit., donde se destacan los avances de este Congreso y de los anteriores.

28 Papel e organizaçâo de magistrados e advogados nas sociedades contemporâneas, IX Congresso Mundial de Direito Judiciário, ed. A.M. Pessoa Vaz, Coimbra, 1995.

29 M. Cappelletti, Algunas reflexiones..., op. cit.. 
La doctrina nacional no ha sido ajena a todo ese revulsivo movimiento de las ideas. Por citar tan sólo algunos hitos: la temprana y anticipatoria producción colectiva La justicia entre dos épocas ${ }^{30}$; sus proyecciones en la dimensión social ${ }^{31}$ y transnacional ${ }^{32}$ y, lo que seguramente constituye la obra más acabada en la que se reflejan las múltiples facetas del más lúcido pensamiento procesal contemporáneo, en proyección hacia el próximo milenio, los Estudios de Derecho Procesal del maestro A.M. Morello ${ }^{33}$.

El vigoroso y comprometido mensaje de Cappelletti, el gran vanguardista cuyo pensamiento preclaro ilumina desde "sus laberintos de tinieblas" 34 éstos por tantas razones complejos e inextricables tiempos finiseculares, tiene asegurada duradera y fructífera continuidad en todas las latitudes. Parece claro que los cultores de la ciencia procesal han comprendido y asumido definitivamente aquella vieja enseñanza de Calamandrei: “...ningún tema como el del proceso merece hoy la atención y el empeño de los estudiosos, porque en ningún campo como en el del proceso es posible encontrar y valorar reunidos, en su angustiosa actualidad todos los aspectos jurídicos, políticos y morales, del problema central de la sociedad humana, que es el problema de la conciliación de la libertad con la justicia" ${ }^{35}$.

30 A. M. Morello, R.O.Berizonce, J.C. Hitrers y C.A. Nogueira, La Justicia entre dos épocas, L.E.P., La Plata, 1983.

31 R.O. Berizonce, Efectivo acceso a la Justicia, con prólogo de M. Cappelletti, L.E.P., La Plata, 1987.

32 J.C. Hitters, Derecho Internacional de los Derechos Humanos, Ediar, Bs.As., 1993, v.I y II. G.L. Sosa., El Derecho Internacional Privado Interamericano y el derecho de la integración, Ed. Tercer Milenio, Bs. As., 1996.

33 A.M. Morello, Estudios de Derecho Procesal. Nuevas demandas. Nuevas respuestas, L.E.P.-Abeledo Perrot, La Plata Bs.As., v.I y II, 1998.

34 A.M. Morello, Reencuentro con Mauro Cappelletti, ed. Fundación Jus, La Plata, 1998 , p.5.

35 P. Calamandrei, Proceso y justicia, en Estudios sobre el proceso civil, EJEA, Bs.As., 1962, trad. S. Sentis Melendo, v.III, p.219. 